\title{
SYNCHROTRON RADIATION: NEW TREND IN X-RAY MAMMOGRAPHY
}

\author{
E. BuRATtini \\ National Laboratory of Nuclear Physics, INFN (National Institute for Nuclear Physics) \\ Via E. Fermi, 00044 Frascati, Rome, Italy \\ and \\ Science Deparment of Verona University, Via Le Grazie, Verona, Italy
}

\begin{abstract}
In order to exploit the peculiar characteristics of synchrotron radiation for X-ray mammography purposes, monochromatic X-ray beams of selected energies from $16 \mathrm{keV}$ to $22 \mathrm{keV}$ have been used for the first time ever to obtain mammograms of surgically removed breast specimens containing cancer nodules. The apparatus devised particularly in view of a possible clinical applications, allowed large breast specimens fixed in space to be exposed to a vertical scanning $\mathrm{X}$-ray beam. The mammograms obtained with synchrotron radiation, compared with those made using a traditional mammographic unit had higher contrast and better resolution demonstrating, in all the cases studied, a high capability to display a large number of structures inside the neoplastic lesions. Thermoluminescent dosimeters were used to determine the doses. The average mean glandular doses, at $17 \mathrm{keV}$ and $18 \mathrm{keV}$, were $1.56 \mathrm{mGy}$ and $0.84 \mathrm{mGy}$, respectively, comparable with the value of $1.41 \mathrm{mGy}$ delivered with the conventional grid apparatus.
\end{abstract}

PACS numbers: 07.85.Qe, 87.59.Ek

\section{Introduction}

It is well known that using conventional X-ray sources, i.e., Coolidge tubes, it is not so easy to detect nodules smaller than $3 \mathrm{~mm}$. Indeed, there are two major causes which reduce the quality of the radiographic images produced by a mammographic conventional unit.

When a breast is exposed to a Mo-anode/Mo-filtered source operating at $30 \mathrm{kV}$, the transmitted beam presents a broad energy band ranging from $14 \mathrm{keV}$ up to $30 \mathrm{keV}$, with a mean value of about $21 \mathrm{keV}$. Only the photons with energy lower than $20 \mathrm{keV}$ - about $50 \%$ - give high radiographic signal of the lesions, the remaining fraction contributes mainly to a strong Compton radiation. Moreover, the radiation is isotropically emitted into the space from a focal spot whose actual dimensions are, at best, larger than $0.4 \times 0.4 \mathrm{~mm}^{2}$ producing geometrical unsharpness. 
On the contrary, synchrotron radiation (SR), especially in the X-ray region, has peculiar characteristics which distinguish it from any other kind of radiation source and enable it to be utilised to obtain high-quality radi. 5 raphs of soft-tissue lesions.

With the aim of overcoming the drawbacks of the Crclidge tube as used in mammography, an intense, monochromatic and quasi-pirillel X-ray beams from the SR Facility, Adone, operating at Frascati, Nationiı Institute for Nuclear Physics (INFN), was used to obtain X-ray mammogran ı: of large surgically excised human breast tissue with enhanced contrast and high $r$ 'solution. In fact, as is well known, previous theoretical studies [1,2] on optimizing mammographic examinations had revealed the existence of an energy range 16- '2.? $\mathrm{keV}$, depending on the breast thickness, able to optimize radiographic images by means of a high signal to noise (S/N) ratio, which allows high contrast and low dos's. These results suggested the use of lower energy intense beams, which are availin.le with suitable intensity only using synchrotron radiation sources.

\section{Synchrotron radiation characteristics}

When relativistic electrons experience centripetal acceleration, due to the presence of a magnetic field, they emit electromagnetic waves which are commonly called "synchrotron radiation" because first observed and experimentally studied, in the visible region, by Elder [3] et al., a research group working in 1947 at the General Electric Research Laboratory (New York), where there was in operation at that time a $70 \mathrm{MeV}$ small synchrotron.

In other words, synchrotron radiation is emitted by electrons or positrons forced to move in a vacuum chamber along a curved trajectory under the action of the Lorentz force, due to the presence of a bending magnetic field. Relativistic particles emit SR in a narrow cone whose geometrical axis is tangent to the instantaneous orbit and lies in the orbital plane. The vertical angular emission, dependent on the particle energy, is very small, usually, fractions of milliradians.

The spectral distribution covers five or more orders of magnitude, typically from the infrared to the X-ray region, and can be characterized via the so-called "critical wavelength" $\lambda_{c}$ which splits the spectrum into two parts of equal radiated power.

The shape of the general function of the synchrotron radiation spectral distribution is shown in Fig. 1; the horizontal wavelength scale is defined in terms of the ratio $\lambda / \lambda_{c}$ and the vertical scale in terms of the number of the photons per second, per $\mathrm{mA}$ of circulating current inside the accelerator, per mrad of horizontal angle, per unitary particles energy, expressed in $\mathrm{GeV}$ and emitted in a relative bandwidth $\Delta \lambda / \lambda=0.1 \%$. It is possible to demonstrate that, to obtain a good spectral flux in the X-ray region, $\lambda_{c}$ must be equal or shorter than $1 \AA$ [4]. This can be achieved with an electron energy of some $\mathrm{GeV}$ and a magnetic field of about $1 \mathrm{~T}$.

Furthermore, synchrotron radiation is $100 \%$ linearly polarized with the direction of the electric field $\boldsymbol{E}$ parallel to the orbital plane. Above and below the plane, the radiation is characterized by an elliptical polarization, due to the pres- 


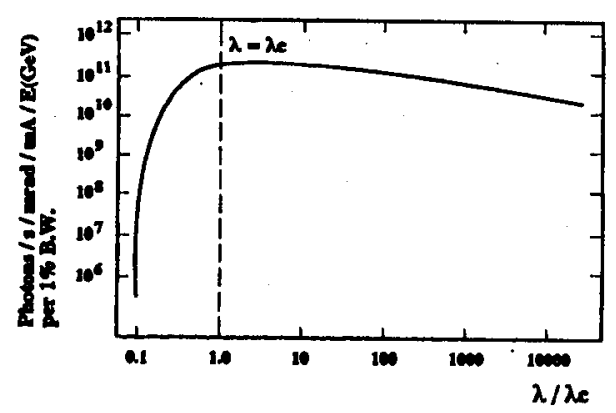

Fig. 1. General function of synchrotron radiation spectral distribution vs. the ratio $\lambda / \lambda_{c}$, where $\lambda_{c}$ is the critical wa velength.

ence of an electrical component perpendicular to the orbital plane which increases with the observation angle.

The peculiar characteristics of synchrotron radiation, such as high intensity, vertical collimation, and polarization, seem to be particularly suitable in radiology. In fact, the high intensity means that crystal monochromators can be used to select the most suitable energy as a function of the radiological task, with a good photon flux up to an energy of several tens of $\mathrm{keV}$. The intrinsic high degree of vertical collimation permits elimination of any geometrical artefacts (unsharpness and magnification).

Finally, the linear polarization could be used to reduce the Compton effect and, consequently, the scattered radiation from the tissue, improving image contrast. Therefore, a synchrotron radiation monochromatic beam may be considered an ideal probe to obtain radiographic images with enhanced contrast and high resolution for early detection of small breast nodules.

\section{Synchrotron radiation imaging system and results}

With the aim of exploiting the potentiality of synchrotron radiation in the field of X-ray mammography, a group of physicists and radiologists has carried out a series of pioneering experiments on the X-ray beam line of the Adone SR Facility. The line collected the photons emitted from a conventional transverse wiggler, installed on a straight section of the $1.5 \mathrm{GeV}$ storage ring Adone, at that time operating in the 3-30 keV energy [5].

The apparatus used to carry out the experiments was equipped with a crystal monochromator and an ionization chamber filled with krypton for monitoring the X-ray beam intensity. A $100 \mathrm{~mm}$ thick beryllium window placed at the end of the line divided the part of the apparatus working under vacuum conditions from that working in air.

Monochromatization was achieved by means of a large single crystal Si (220) mounted on a high-precision goniometer to select the most suitable wavelength for Bragg diffraction. The monochromator was installed on a horizontal table equipped with three-axis, $X Y Z$ movements, all driven by step motors and controlled by PLCs. As shown in Fig. 2, the $Y-Z$ movements allowed the goniometer rotation axis to be placed in the correct position with respect to the $\mathrm{X}$-ray beam axis 


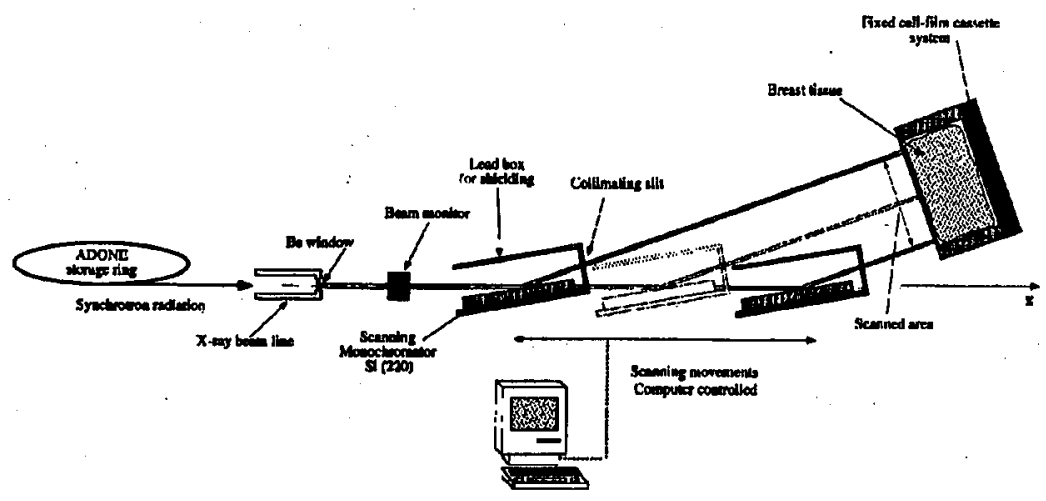

Fig. 2. Imaging system apparatus.

propagation; moving the goniometer along the longitudinal $X$-direction, it was possible to vertically scan the beam reflected by the crystal.

With this apparatus, mammograms can be performed by simply moving the monochromator along the $X$-axis and placing the sample jointly with the imaging system, fixed in space, orthogonal to the direction of the monochromatic beam.

The size of the area covered during the scan was, horizontally, function of the monochromator geometrical acceptance and, vertically, function of the maximum run along the $X$-movement. In the present case, the dimensions of the crystal reflecting surfaces were $70 \times 30 \mathrm{~mm}^{2}$ and the maximum excursion of the longitudinal movement was $300 \mathrm{~mm}$ and working in the energy range from 17 to $18 \mathrm{keV}$, it was possible to cover about $70 \times 100 \mathrm{~mm}^{2}$ on the cassette plane. The scan speed along the $X$-axis was optimizable as a function of the $\mathrm{X}$-ray beam intensity and the specimen thickness making it possible to predetermine and control the exposure.

The specimens were placed in perspex cells designed to accommodate diverse sizes in order to avoid specimen displacement when the cells were moved jointly with the film cassette.

For the comparison, the same part of human breast, excised surgically from living patient for the presence of neoplastic lesions, was placed first in front of a conventional source, then in front of an optimized synchrotron radiation beam. The conventional one was a MSM - General Medical Merate - having a rotating anode with molybdenum anticathode and filter, microfocussing variable from 0.1 or 0.3 , anti-scattering grid (ratio 5), and accelerator voltage working between 22 and $35 \mathrm{kV}$.

The detecting system used for both techniques consisted of cassette, intensifying screen, and dedicated developer. Care was taken not to change the projection angle.

After preliminary experiments made with a different technique $[6,7]$, several mammograms were obtained of phantoms and of large breast parts, both fixed in space, using the scanning technique.

In all the cases analyzed the radiological content of the synchrotron radiation mammograms was clearly superior in contrast and resolution, which are, as is well 

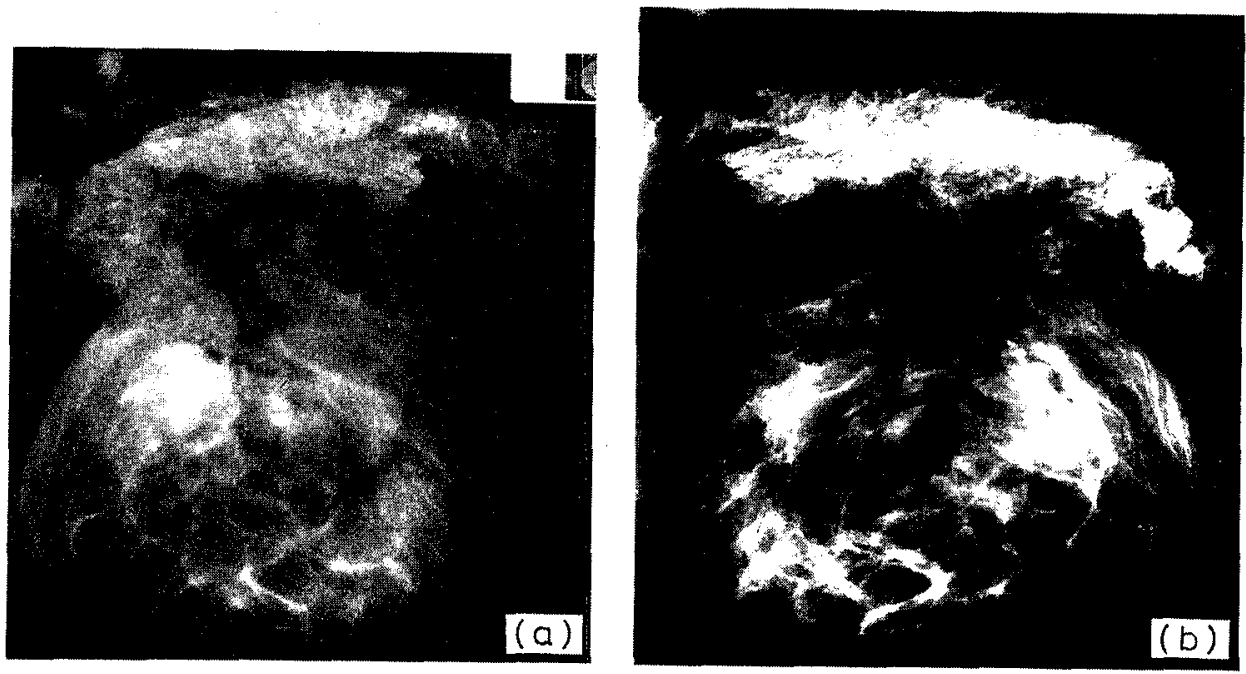

Fig. 3. Radiographs of the same surgically excised breast specimen obtained (a) with conventional mammographic unit; (b) with synchrotron radiation using vertically scanning beam technique.

known, important characteristics in the detection of radiopaque zones and the identification of the structures inside them. In Fig. 3 where a post-surgery nodule, excised to a patient suffering for a fibrocis ic mastopaty and radiographed using a conventional source (a) and SR (b) is shown, it is clear that the nodule structure is more evident and better resolved in SR case.

The best results on both phantoms and breast specimens were obtained with an energy beam between 17 and $18 \mathrm{keV}$, depending on the specimen thickness. The comparison of the two series of radiographs was invariably carried out as a double "blind analysis", confirming unequivocally that the best radiographs were produced with the synchrotron radiation beam.

Finally, to exploit the high degree of vertical collimation typical of a synchrotron radiation beam, several mammograms were carried out, separating the cassette from the cell-specimen and changing the distance from 0 to $50 \mathrm{~cm}$. The radiological quality of the image obtained separating the cassette from the specimen is higher due to a strong reduction of the scattered radiation, which is the main cause of the poor quality of mammograms made with a conventional unit.

The impressive reduction of scattered radiation achievable by separating the breast tissue from the cassette can be compared with the effect present when a "pre-post breast slit device" is used for scatter reduction in dual-slit mammography. However, using a synchrotron radiation beam, reduction in beam intensity is eliminated.

\section{Dose evaluation}

The doses delivered by conventional and synchrotron radiation mammograms were determined using a BR12 dosimetric phantom [8]. BR12 has X-ray properties similar to a mixture of $50 \%$ adipose and $50 \%$ glandular tissue by weight. 
The phantom consisted of five square slabs $\left(10 \times 10 \times 1 \mathrm{~cm}^{3}\right)$ lying one on top of the other. Each slab had a cavity for positioning five LiF detectors (TLD-100). The thermoluminescent detectors, individually labelled, were calibrated in the range of equivalent energies from 13 to $25 \mathrm{keV}$ with a low-energy standard chamber [9]. All the TLD readings were processed by subtracting the average reading of a group of unexposed dosimeters of the same set and multiplying the results by the calibration factor previously described.

The dosimetric phantom was exposed to a conventional X-ray beam and to monochromatic synchrotron radiation using the exposure parameters of a properly exposed radiograph (optical density as close as possible to 1.2) of the computerized imaging reference system (CIRS) standard test phantom. The phantom, made of an epoxy material designed to match the density and attenuation properties of breast tissue, was $5 \mathrm{~cm}$ thick and had the same X-ray transmission as an average breast.

Table gives the values of entrance exposure $\left(X_{\mathrm{e}}\right)$, entrance dose in BR12 $\left(D_{\mathrm{e}}\right)$, and mean glandular dose to a $5 \mathrm{~cm} \mathrm{BR12}$ phantom $\left(D_{\mathrm{g}}\right)$.

TABLE

Entrance exposure (with backscatter), BR12 entrance dose, and mean glandular dose to a BR12 $5 \mathrm{~cm}$ thick phantom delivered by synchrotron radiation and conventional unit mammography.

\begin{tabular}{l|c|c|c}
\hline \hline $\begin{array}{l}\text { Imaging } \\
\text { source }\end{array}$ & $\begin{array}{c}\text { Entrance } \\
\text { exposure } X_{\mathrm{e}}\end{array}$ & $\begin{array}{c}\text { Entrance } \\
\text { dose } D_{\mathrm{e}}\end{array}$ & $\begin{array}{c}\text { Mean glandular } \\
\text { dose } D_{\mathrm{g}}\end{array}$ \\
\hline $\begin{array}{l}\text { Synch. rad. } \\
(17 \mathrm{keV})\end{array}$ & $2.29 \times 10^{-4} \mathrm{C} / \mathrm{kg}$ & $5.85 \mathrm{mGy}$ & $1.56 \mathrm{mGy}$ \\
$\begin{array}{l}\text { Synch. rad. } \\
(18 \mathrm{keV})\end{array}$ & $1.05 \times 10^{-4} \mathrm{C} / \mathrm{kg}$ & $2.68 \mathrm{mGy}$ & $0.837 \mathrm{mGy}$ \\
$\begin{array}{l}\text { Conventional } \\
\text { no-grid (26 kV) } \\
\text { Conventional } \\
\text { grid (28 kV) }\end{array}$ & $1.31 \times 10^{-4} \mathrm{C} / \mathrm{kg}$ & $3.35 \mathrm{mGy}$ & $0.696 \mathrm{mGy}$ \\
\end{tabular}

\section{Conclusions}

To the authors' knowledge this is the first time that an X-ray source other than a Coolidge tube has been used in image diagnostics for X-ray mammography. The images obtained using a "scanning beam" technique, employable in human in vivo applications, have demonstrated without any doubt that synchrotron radiation mammography has an impressive capability to display a large number of structures inside a neoplastic lesion, with high resolution and enhanced contrast. The mean dose delivered by a $17 \mathrm{keV}$ monochromatic beam is almost the same as the dose from the conventional grid technique. Increasing the energy to $18 \mathrm{keV}$, the mean dose decreases significantly $(-46 \%)$, the radiographic contrast decreases 
very little, but the image quality is still better than that of conventional grid mammography.

\section{References}

[1] R.J. Jenning, R.J. Eastgate, M.P. Siedband, D.L. Ergun, Med. Phys. 8, 629 (1981).

[2] M.W. Ragozzino, Med. Phys. 9, 493 (1982).

[3] F.R. Elder, A.M. Gurewitsch, R.V. Langmuir, H.C. Pollock, Phys. Rev. 71, 828 (1947).

[4] E.E. Koch, in: Synchrotron Radiation, Vol. 1a, Eds. D.E. Eastman, Y. Farge, North-Holland, Amsterdam 1983, p. 15.

[5] E. Burattini, A. Reale, E. Bernieri, N. Cavallo, A. Morone, M.R. Masullo, R. Rinzivillo, G. Dalba, P. Fornasini, C. Mencuccini, Nucl. Instrum. Methods 208, 91 (1983).

[6] E. Burattini, M. Gambaccini, O. Rimondi, P.L. Indovina, M. Pocek, G. Simonetti, M. Benassi, C. Tirelli, R. Passariello, Rev. Sci. Instrum. 63, 638 (1992).

[7] G. Simonetti, M. Pocek, E. Burattini, M. Gambaccini, E. Cossu, G.B. Grassi, Le Sein 2, 4 (1992).

[8] R.C. Speiser, E.M. Zanrosso, L.S. Jeronim, Br. J. Radiol. 13, 667 (1977).

[9] L. Stanton, T. Villafana, L.J. Day, D.A. Lightfood, Radiology 150, 577 (1984). 